\title{
Retrograde Type A Aortic Dissection after Thoracic Endovascular Aortic Repair for Type B Dissection
}

\author{
Masato Hayakawa, MD, ${ }^{1}$ Takaaki Nagano, $\mathrm{MD},{ }^{2}$ Isao Nishijima, MD,${ }^{3}$ Kento Shinzato, MD, ${ }^{1}$ \\ Ryo Ikemura, MD,${ }^{1}$ Kazufumi Miyagi, MD,${ }^{1}$ Kiyoshi Iha, MD, PhD,${ }^{1}$ Shigenobu Senaha, MD,${ }^{3}$ \\ Mitsuyoshi Shimoji, MD, ${ }^{3}$ Mitsuru Akasaki, $\mathrm{MD}^{3}$ \\ ${ }^{1}$ Department of Cardiovascular Surgery, Chubu Tokushukai Hospital, Kitanakagusuku, Japan; ${ }^{2}$ Department of Thoracic and \\ Cardiovascular Surgery Graduate School of Medicine, University of the Ryukyus, Nishihara, Japan; ${ }^{3}$ Department of Cardiovascular \\ Surgery, Nanbu Tokushukai Hospital, Yaese, Japan
}

\section{ABSTRACT}

Background: A 64-year-old woman presented with dilatation of the distal aortic arch secondary to chronic type B aortic dissection.

Case Report: The patient underwent fenestrated thoracic endovascular aortic repair (TEVAR) for closure of the entry site, and reconstruction of the left subclavian artery with a covered stent. On the 40th postoperative day, a retrograde type A aortic dissection (RTAD) was observed on computed tomography and she underwent emergency surgery. The entry tear, related to the proximal bare metal stent, was located in front of the aortic arch. A partial aortic arch replacement was performed.

Conclusion: Consideration of the risk factors of RTAD is important when performing TEVAR.

\section{INTRODUCTION}

Thoracic endovascular aortic repair (TEVAR) has been increasingly used in the treatment of thoracic aortic aneurysms and dissections [Kuwano 2012]. However, one of the most feared complications of this procedure is retrograde type A aortic dissection (RTAD), which has a low incidence but high mortality rate [Dong 2009]. We report a case of retrograde type A aortic dissection after TEVAR for chronic type $\mathrm{B}$ aortic dissection and review the causes and management of this complication of TEVAR.

\section{CASE REPORT}

A 64-year-old woman complaining of sudden chest back pain was admitted to our emergency department.

Received April 19, 2020 ; accepted fune 12, 2020.

Correspondence: Masato Hayakawa, MD, Department of Cardiovascular Surgery, Chubu Tokushukai Hospital, 801 Higa, Kitanakagusuku, Nakagami, Okinawa, 901-2393 Japan; +81-98-932-1110; fax: +81-98-923-1659 (e-mail:yunta_sp@mail.goo.ne.jp).
Contrast-enhanced CT showed an acute type B aortic dissection, but there was no sign of organ malperfusion, so she was managed by optimal medication. A CT scan on the 15 th day after the onset of her symptoms revealed that the maximal diameter of the distal aortic arch had expanded from $38 \mathrm{~mm}$ to $44 \mathrm{~mm}$ (Figure 1, A). She was scheduled for elective zone 2 TEVAR with left subclavian artery (LSCA) revascularization 25 days after onset to close the entry.

Under general anesthesia, we first constructed the fenestrated stent graft with Relay Plus $32 \mathrm{~mm} \times 28 \mathrm{~mm} \times 250$ mm (Bolton Medical, Sunrise, FL, USA). A 7-mm hole was made between the first and second stents by using a cautery system, and to prevent a type III endoleak AZUR35 $6 \mathrm{~mm} \times$ $20 \mathrm{~cm}$ (Terumo, Tokyo, Japan) was fixed with a Nespyrene 6-0 suture to the edge of the fenestration (Figure 1, B). We placed Relay Plus $26 \mathrm{~mm} \times 26 \mathrm{~mm} \times 155 \mathrm{~mm}$ (Bolton Medical, Sunrise, FL, USA) in the descending aorta at the Th10 level, and the fenestrated stent graft was then placed from the distal left common carotid artery (LCCA) into the descending aorta, via the right common femoral artery (RCFA). Gore VIABAHN VBX $8.0 \mathrm{~mm} \times 39 \mathrm{~mm}$ (W.L. Gore and Associates, Arizona, USA) was deployed in the LSCA orifice to reconstruct the LSCA (Figure 1, C). Aortography showed

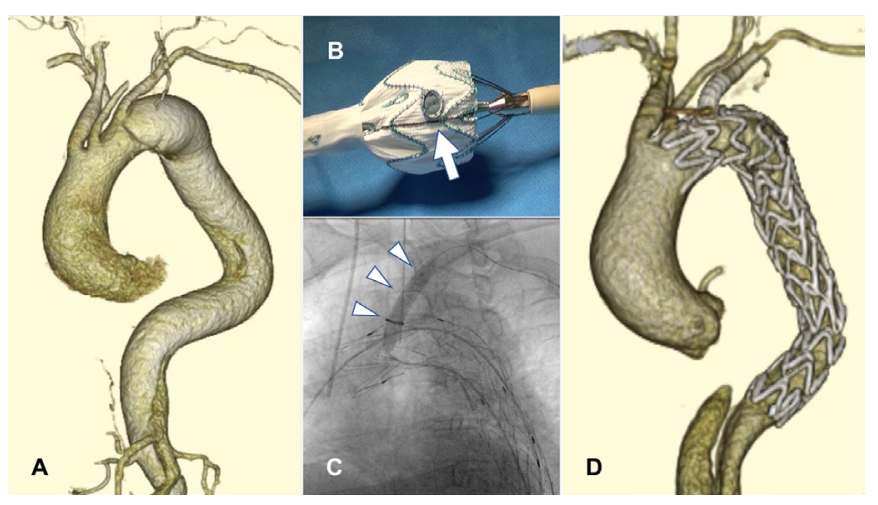

Figure 1. A, Preoperative three-dimensional computed tomography (3-D CT) showed type B aortic dissection. B, We made a fenestrated stent graft with Relay Plus and AZUR 35 was fixed to the edge of the fenestration (arrow). C, Left subclavian artery was reconstructed using Gore VIABAHN VBX (arrowhead). D, Postoperative 3-D CT showed successful fenestrated TEVAR. 


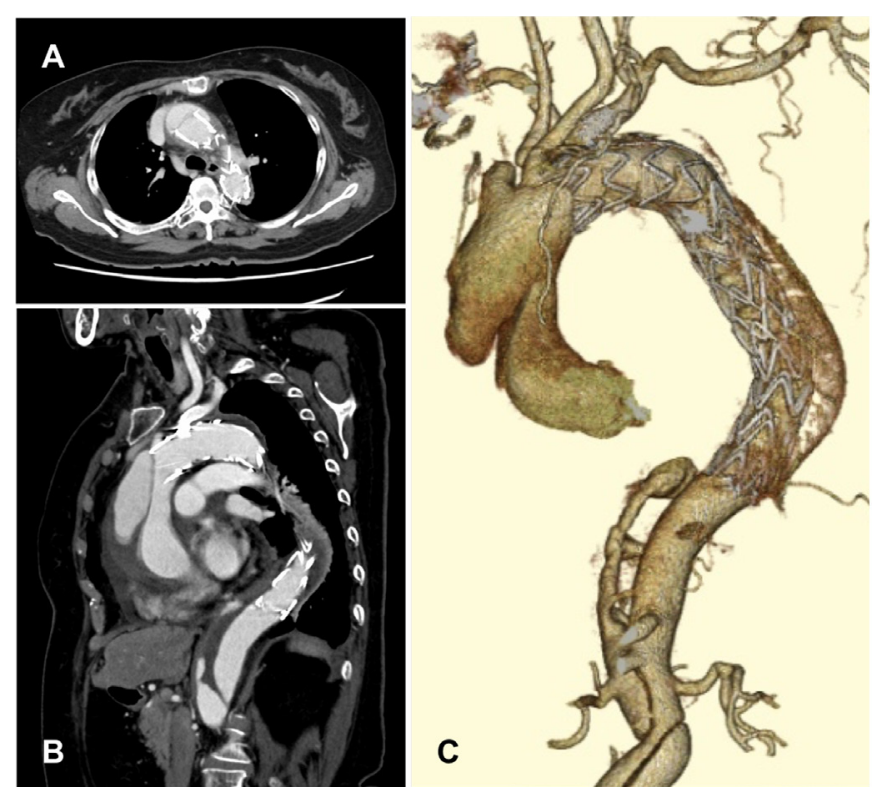

Figure 2. Contrast-enhanced CT revealed stent graft-induced retrograde type A aortic dissection (RTAD).

type Ia endoleak into the false lumen; therefore, we performed balloon dilatation of only the covered stent and did not dilate the stent graft, and the final aortography revealed no endoleak.

The patient had an uneventful recovery and was discharged on the 21st postoperative day (Figure 1, D). However, she returned to our hospital with general malaise on the 40th postoperative day. Contrast-enhanced CT revealed an acute type A aortic dissection, with the right brachiocephalic artery occluded by the false lumen (Figure 2). She was diagnosed with retrograde type A aortic dissection (RTAD) and underwent emergency surgery.

We performed emergency open surgery through a median sternotomy. Cardiopulmonary bypass was established by placing an arterial cannula through the right common femoral artery and a venous cannula through the right atrium. Under circulatory arrest with hypothermia at $23^{\circ} \mathrm{C}$, the ascending aorta was opened and selective antegrade cerebral perfusion was started. The entry tear related to the proximal bare metal stent was located in front of the aortic arch (Figure 3, A). After ligation and disconnection of the LCCA, the entry tear was excised and the distal margin of the native aorta was reinforced with Teflon felt in a sandwich fashion. The stent graft did not need to be cut because the bare stent tip was narrowed with a tourniquet during the distal stump formation (Figure 3, B). J Graft $24 \mathrm{~mm}$ four-branch (Japan Lifeline, Tokyo, Japan) was anastomosed to the distal portion with felt reinforcement, as described above, but not to the stent graft. After reconstructing the LCCA, the graft was anastomosed to the proximal aorta at the sinotubular junction. Finally, the branch graft was anastomosed to the right brachiocephalic artery.

After the operation, the patient experienced left hemiplegia and became unconscious. A head CT showed

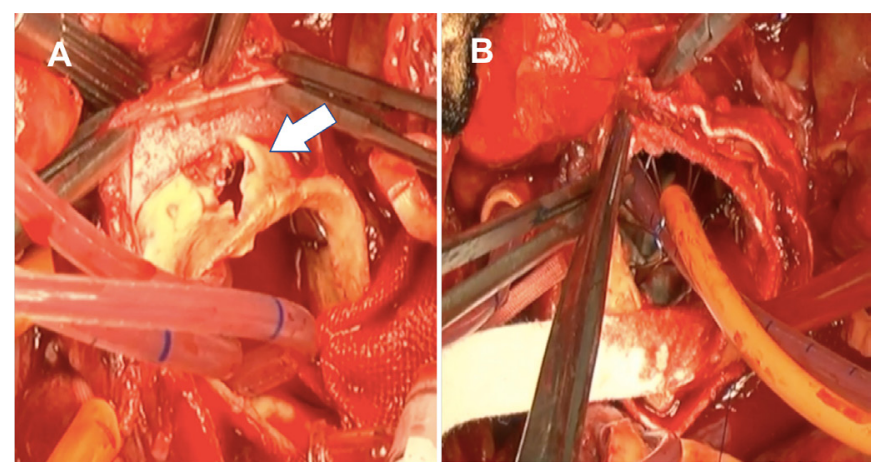

Figure 3. A, Intraoperative view of the transected ascending aorta showed the entry related to the proximal bare metal stent (arrow). B, The distal anastomosis was performed while shrinking the proximal bare metal stent.

extensive cerebral infarction in the right cerebral hemisphere. However, her level of consciousness gradually improved, and she was extubated on the 11th postoperative day. On the 35 th postoperative day, she was transferred to a rehabilitation hospital, from which she was discharged six months after the operation, and spent her time at home. One year after the operation, her level of consciousness was clear and, although left paralysis of the lower extremities was observed, she was able to walk with an orthosis attached.

\section{DISCUSSION}

In recent years, thoracic endovascular aortic repair (TEVAR) has been applied to the treatment of aortic dissection due to its low invasiveness, usefulness, and good results. However, there have been reports of retrograde type A aortic dissection (RTAD) after TEVAR. RTAD is a life-threatening complication that requires emergency surgery [Dong 2009].

The incidence of RTAD after TEVAR ranges from $1.33 \%$ [Eggebrecht 2009] to 2.5\% [Chen 2017] and its mortality rate ranges from 37.1\% [Chen 2017] to 40\% [Eggebrecht 2009]. Eggebrecht and colleagues report that the incidence of RTAD was $46 \%$ within 30 days after TEVAR and $31 \%$ three months later [Eggebrecht 2009]. On the other hand, there have been reports of onset of RTAD during TEVAR [Canaud 2014], and there is no certain tendency regarding the onset time. In our case, RTAD was considered to have developed 37 days after TEVAR.

Risk factors for RTAD are reported in terms of (1) indication for TEVAR, (2) device factors, and (3) technical factors at the time of TEVAR. First, regarding the indications for TEVAR, aortic dissection is more likely to develop RTAD than a true aortic aneurysm, and an acute aortic dissection is more likely to develop than a chronic one [Dong 2009; Eggebrecht 2009; Chen 2017; Canaud 2014]. Next, as a device factor, the risk of RTAD is higher in patients treated with proximal bare stent, [Chen 2017; Ehrlich 2008; Victor 2016] and in those with stent graft oversizing [Chen 2017; Canaud 2014]. Finally, as a technical factor, the deployment of the stent graft in the proximal landing zone (zone 0-2) [Eggebrecht 
2009; Chen 2017; Canaud 2014] and the post-ballooning of the stent graft are risk factors for RTAD [Eggebrecht 2009]. In our case, a stent graft with a proximal bare stent was used for the chronic aortic dissection, and the proximal landing was zone 2. The ratio of oversize on the proximal side of the stent graft was $3.2 \%$ to $14.2 \%$. Although post-ballooning to the covered stent used for LSCA reconstruction was performed, balloon compression was not performed on the stent graft main body. The second intraoperative finding showed longitudinal entry from the tip of the proximal bare stent toward the ascending aorta. For this reason, in our case, entry probably occurred due to the proximal bare stent digging into the fragile aortic wall, causing the RTAD to develop.

The gold standard of surgical management for patients with RTAD is graft replacement of the ascending aorta with or without hemi- or total arch replacement [Erlich 2008; Shuyang 2012]. Among them, several options for stump formation at the distal anastomosis have been reported, including a method of direct anastomosis to the stent graft [Victor 2016], a method of inserting and fixing a short elephant trunk into the stent graft, and the use of an open stent graft [Shuyang 2012]. In our case, the stent graft was fenestrated and the LSCA was reconstructed with a covered stent, so it was desirable to avoid the insertion of a short elephant trunk or open stent graft. Further, even if proximal bare stents or a part of the stent graft is cut off, the distance to the fenestration is short and it is difficult to secure the optimal distance for anastomosis. Fortunately, intraoperative findings indicated that entry occurred from the distal end of the bare stent, so entry resection was possible if the distal site of the aorta was trimmed at the level of the distal end of the bare stent. For this reason, after the entry was resected, a gap was created between the bare stent and the aortic wall by narrowing the bare stent tip with a tourniquet, and a distal stump was formed using the gap as an anastomotic portion, and then the graft could be anastomosed. The primary lesson of this case is that RTAD after fenestrated TEVAR with branch reconstruction may make it difficult to form a stump at the distal anastomosis. A further lesson is that better clinical results could have been obtained if, in addition to the femoral artery cannula, the right axillary artery was also cannulated to enhance cerebral perfusion when establishing the cardiopulmonary bypass. Regarding this point, we will make use of this experience in the future.

\section{CONCLUSION}

We experienced a case in which RTAD developed after TEVAR for chronic type B aortic dissection. It is necessary to understand the risk factors of RTAD when TEVAR is performed, and it is necessary to devise the distal anastomosis when RTAD occurs.

\section{REFERENCES}

Canaud L, Ozdemir BA, Patterson BO, et al. 2014. Retrograde aortic dissection after thoracic endovascular aortic repair. Ann Surg 260:389-95.

Chen Y, Zhang S, Liu L, et al. 2017. Retrograde type A aortic dissection after thoracic endovascular aortic repair: A Systematic Review and MetaAnalysis. J Am Heart Assoc 6:e004649.

Dong ZH, Fu WG, Wang YQ, et al. 2009. Retrograde type A aortic dissection after endovascular stent graft placement for treatment of type B dissection. Circulation 119:735-41.

Eggebrecht H, Thompson M, Rousseau H, et al. 2009. Retrograde ascending aortic dissection during or after thoracic aortic stent graft replacement: insight from the European registry on endovascular aortic repair complications. Circulation 120:S276-81.

Ehrlich PM, Nienaber AC, Rousseau H, et al. 2008. Short-term conversion to open surgery after endovascular stent-grafting of the thoracic aorta: the Talent thoracic registry. J Thorac Cardiovasc Surg 135:1322-6.

Kuwano H, Amano J, Yokomise H, et al. 2012. Thoracic and cardiovascular surgery in Japan during 2010: annual report by The Japanese Association for Thoracic Surgery. Gen Thorac Cardiovasc Surg 60:680-708.

Shuyang L, Hao L, Chunsheng W, et al. 2012. Surgical treatment for retrograde type A aortic dissection after endovascular stent graft placement for type B dissection. Interact Cardiovasc Thoracic Surg 14:538-42.

Victor XM, Milagros M, Daniel FM, et al. 2016. Repair of late retrograde type A aortic dissection after TEVAR: Causes and management. J Card Surg 31:164-7. 